\title{
Contribución del EPCP al mercadeo de las papas nativas en los Andes
}

\author{
G. López ${ }^{1}$, R. Oros ${ }^{2}$, F. Rodríguez ${ }^{3}$, J. López ${ }^{4}$, K. Manrique ${ }^{5}$ \\ RESUMEN
}

El Enfoque Participativo en Cadenas Productivas (EPCP) es una metodología que en los últimos nueve años ha contribuido de manera significativa al reposicionamiento de las papas nativas en los mercados urbanos de los países andinos. Mediante la convocatoria y articulación de los diferentes actores de la cadena productiva se han logrado introducir a ciudades como Lima, La Paz, Cochabamba, Santa Cruz, Riobamba, entre otras, las papas nativas, tanto frescas y seleccionadas como en hojuelas (chips), con nombres de marca y en diferentes presentaciones. En este sentido, podemos afirmar que la visibilidad que han tenido las papas nativas ha sido el resultado de un emprendimiento colectivo, entendiendo como tal a un conjunto de actores que actúan y colaboran en un ambiente de confianza y aprendizaje mutuo, alrededor de "cosas" concretas (los productos, las marcas) que capitaliza el trabajo del grupo. Por otro lado, la experiencia ha demostrado que las aplicaciones del EPCP no se limitan solo a la realización de negocios o encadenamientos comerciales, sino que se prolonga mucho mas allá, constituyendo un espacio o plataforma donde los diferentes actores continúan realizando actividades en beneficio de la cadena, promoviendo el producto y beneficiando a los pequeños productores rurales.

Palabras clave: papas nativas, cadenas productivas de alimentos.

\section{Contribution of PMCA to the marketing of native potato varieties in the Andes}

\begin{abstract}
The participatory market chain approach (PMCA) is a methodology that has contributed significantly to positioning the native potato varieties in urban markets in Andean countries. By calling and articulating different stakeholders of the potato market chain, it has been possible to introduce fresh tubers and colored chips of native potato varieties in urban markets of Lima, La Paz, Cochabamba, Santa Cruz and Riobamba, with commercial brands and different presentations. It can be concluded that these products owe their visibility to collective action by PMCA participants. By interacting and cooperating in the PMCA process, that favors trust and learning, these participants have worked together towards concrete objectives. The experience shows that PMCA applications are not only limited to identifying business opportunities and tapping into them. They also generate stakeholder platforms. These platforms serve as coordinating instances where stakeholders can continue interacting for the benefit of small scale farmers.
\end{abstract}

Key words: Native Potato, food production chain.

\footnotetext{
${ }^{1}$ Iniciativa Papa Andina del Centro Internacional de la Papa, Lima - Perú.

${ }^{2}$ Fundación PROINPA, Cochabamba - Bolivia.

${ }^{3}$ Fundación PROINPA, Cochabamba - Bolivia.

${ }^{4}$ Fundación MARCO, Riobamba - Ecuador.

${ }^{5}$ Proyecto INCOPA, del Centro Internacional de la Papa, Lima - Perú.
} 


\section{Introducción}

Cuando se hizo el primer estudio de mercado sobre papas nativas (especies nativas de Solanum tuberosum subsp. andigenum) en la región andina, en Perú, uno de los países con mayor diversidad y consumo, se pudo apreciar la necesidad de modernizar elementos de la presentación comercial como la calidad del producto y del empaque y la necesidad de realizar una mayor difusión de las cualidades intrínsecas de la mayoría de variedades, para incrementar la demanda y el precio. Había una brecha entre el conocimiento y el consumo debido a que las amas de casa encontraban las papas nativas sucias, en mal estado, teniendo que descartar una porción importante de la compra realizada. El estudio fue un esfuerzo conjunto entre una entidad del Estado (PRONAMACHS), una entidad no gubernamental (PRISMA ONGD) y el Centro Internacional de la Papa (CIP) a través del Proyecto Papa Andina. A partir de este estudio se identificaron nuevas rutas de comercialización (papas nativas frescas y seleccionadas y hojuelas), diferentes a la venta tradicional.

Las papas nativas fueron identificadas como uno de los productos promisorios que tenían los pequeños productores alto andinos en situación de extrema pobreza, la gran mayoría de estas variedades se producen en forma orgánica, debido a que la pobreza limita su acceso a productos químicos (fertilizantes, plaguicidas, entre otros), ello se veía como una debilidad. La estrategia interinstitucional fue convertir esta debilidad en fortaleza en los espacios urbanos, donde lo natural empezaba a tener una mayor valoración. Con la globalización, a este aspecto, se suman temas como la biodiversidad y pluriculturalidad, que en las próximas décadas serán considerados de mayor importancia debido a las condiciones del contexto mundial. El objetivo de este trabajo fue identificar, validar y promover un producto promisorio común en las diversas comunidades altoandinas con el propósito de incrementar los ingresos de las familias, aliviando la pobreza y contribuyendo al mejoramiento de la calidad de vida de las personas.

\section{Materiales y Métodos}

Una vez que se identificó el producto "papas nativas" como el que se debería promover para llevar los beneficios de manera directa a las comunidades alto andinas, y su desarrollo comercial en las ciudades como una de las actividades fundamentales para obtener beneficios económicos (mayor precio) y sociales (levantamiento del autoestima), se buscó una metodología que fuera participativa e inclusiva, tomando el RAAKS (Rapid Appraisal of Agricultural Knowledge Systems) (Engel, 2003), con un enfoque de cadena productiva, el cual se convirtió luego en el Enfoque Participativo de Cadenas Productivas (EPCP) (Thiele y Bernet, 2005), que permitió desarrollar el mercado de las papas nativas, no solo en Perú, sino también en otros países de la región andina como Bolivia y Ecuador.

El EPCP fue más que una metodología para desarrollar mercados, porque las actividades participativas no solo permitieron la promoción comercial de las papas nativas y su difusión en los mercados urbanos de las grandes ciudades, sino que se constituyo en un medio de intercambio de conocimientos, de espacio para formar y consolidar capital social y para impulsar la acción colectiva. Pero uno de los principales beneficios reconocidos por los participantes es la capacidad de concretizar las ideas, es decir, que se obtiene de la aplicación de los procesos EPCP “cosas" concretas, productos tangibles, que pueden ser tocados, vistos, consumidos, con nombres (marcas) y que se venden en los mercados. Los productores y todos los involucrados manifestaron al momento de participar en los diversos procesos de aplicación de la metodología, que estaban cansados de reuniones y planes de trabajos, que querían cosas concretas, que el tiempo invertido no fuera algo intangible, sino un producto concreto, cosa que ven con el EPCP. La diferencia del EPCP con otras metodologías vinculadas a las cadenas productivas es que llega a la implementación participativa de las oportunidades identificadas y no se queda solamente en la formulación de una estrategia de intervención que es entregada a los actores (Bernet et al., 2006) Las ideas analizadas se llevan a la práctica. 


\section{Resultados y discusión}

Así tenemos en Perú a Tikapapa (papa fresca seleccionada, limpia y envasada) y Papy Bum (chips de papas nativas amarillas) en un primer momento, y luego a toda la variedad de marcas que han salido al mercado limeño (Lays, Papas Nativas, entre otros); en Bolivia a "Que rica Papa" y "Lucana" (papas frescas seleccionadas y chips respectivamente), y luego también a las diferentes marcas de papas nativas que se han introducido en el mercado de La Paz, Cochabamba y Santa Cruz. Y recientemente en Ecuador tenemos a la marca "Papas Nativas" (papa fresca seleccionada) generada en Riobamba, también a partir de un proceso EPCP. Cabe señalar que pasar de las primeras marcas (como sucedió en Perú con el caso de las hojuelas Papy Bum) a una mayor cantidad de marcas de papas nativas con valor agregado (siguiendo con el ejemplo de Perú ahora hay hojuelas producidas por grandes empresas que se han interesado en el negocio incorporando el criterio de responsabilidad social empresarial) no fue un resultado inmediato, sino el resultado de un proceso de ocho o nueve años de trabajo continuo.Conforme señalamos anteriormente, el EPCP no solo contribuyo al desarrollo del mercado sino haciendo inclusivo el proceso, propiciando que los productores intervengan activamente, y mediante la articulación con otros actores de la cadena (procesadores, comercializadores, etc.) y lo mas importante, los resultados obtenidos en todos los procesos, en los tres países andinos, no fue el resultado de un empresario individual, sino fue el resultado de una acción colectiva, donde participaron diferentes actores (agricultores asociados e independientes, comerciantes mayoristas, procesadores, entidades de desarrollo gubernamentales y no gubernamentales, entre otros), concertado (buscando todos un objetivo común que les permita satisfacer sus interés particulares), inclusivo (tomando a los agricultores como el actor principal a quien beneficiar), realista y sostenible (trabajando en función a demandas del mercado) y con una proyección social, económica y política importante, donde no todo es solo el negocio, sino que el esfuerzo mas allá de lo puramente comercial, constituyéndose —en muchas de las experiencias - plataformas multiactores para dar continuidad a las innovaciones (Devaux et al, 2009).

\section{Conclusiones}

A partir de las "cosas" (productos concretos o marcas) generadas por el EPCP, los actores empiezan a interactuar en función al conocimiento, la confianza y la colaboración, surgiendo la necesidad de continuar las relaciones personales, comerciales e institucionales establecidas, conformando Plataformas de Concertación multiactores donde dan continuidad a los negocios emprendidos colectivamente, surgen nuevas iniciativas, tienen una mayor vinculación con el sector institucional publico y privado y sobre todo empiezan a realizar actividades de incidencia política ante los organismos locales, regionales y nacionales. Las ideas hechas realidad, son la expresiones mas concreta de las innovaciones comerciales, tecnológicas e institucionales, alrededor de las cuales se focalizan los intereses, las investigaciones y los trabajos de los actores que participan en el proceso.

\section{Literatura citada}

Bernet, T., Thiele, G., Zschocke, T. 2006. Participatory Market Chain Approach (PMCA) User Guide. Primera Edición. Centro Internacional de la Papa (CIP) Lima, Perú. 169 p.

Devaux, A., Douglas, H., Velasco, C., Thiele, G., López, G., Bernet, T., Reinoso, I., Ordinola, M. 2009. Collective action for market chain innovation in the Andes. Food Policy 34(1): 3138.

Engel, P., Salomon, M. 2003. Facilitating Innovation for Development: A RAAKS Resource Box. Amsterdam. 400 p.

Thiele, G., Bernet, T. 2005. Conceptos, Pautas y Herramientas: Enfoque Participativo en Cadenas Productivas y Plataformas de Concertación. Primera Edición. CIP, Lima - Perú. 171 p. 